\title{
On some complex differential and difference equations concerning sharing function
}

Hua Wang ${ }^{1 *}$, Lian-Zhong Yang ${ }^{2}$ and Hong-Yan Xu ${ }^{1}$

"Correspondence: hhhlucy2012@126.com

1 Department of Informatics and Engineering, Jingdezhen Ceramic Institute, Jingdezhen, Jiangxi 333403, P.R. China

Full list of author information is available at the end of the article

\begin{abstract}
By using the theory of complex differential equations, the purpose of this paper is to investigate a conjecture of Brück concerning an entire function $f$ and its differential polynomial $L(f)=a_{k}(z) f^{(k)}+\cdots+a_{0}(z) f$ sharing a function $\alpha(z)$ and a constant $\beta$. We also study the problem on entire function and its difference polynomials sharing a function.
\end{abstract}

MSC: 39A50; 30D35

Keywords: entire function; Brück's conjecture; difference equation

\section{Introduction and main results}

Let $f$ be a nonconstant meromorphic function in the whole complex plane $\mathbb{C}$. We shall use the following standard notations of the value distribution theory:

$$
T(r, f), \quad m(r, f), \quad N(r, f), \quad \bar{N}(r, f),
$$

(see Hayman [1], Yang [2] and Yi and Yang [3]). We denote by $S(r, f)$ any quantity satisfying $S(r, f)=o(T(r, f))$, as $r \rightarrow+\infty$, possibly outside of a set with finite measure. A meromorphic function $a(z)$ is called a small function with respect to $f$ if $T(r, a)=S(r, f)$. In addition, we will use the notation $\sigma(f)$ to denote the order of meromorphic function $f(z)$, and $\tau(f)$ to denote the type of an entire function $f(z)$ with $0<\sigma(f)=\sigma<+\infty$, which are defined to be (see [1])

$$
\sigma(f)=\limsup _{r \rightarrow \infty} \frac{\log T(r, f)}{\log r}, \quad \tau(f)=\limsup _{r \rightarrow \infty} \frac{\log M(r, f)}{r^{\sigma}} .
$$

We use $\sigma_{2}(f)$ to denote the hyper-order of $f(z), \sigma_{2}(f)$ is defined to be (see [3])

$$
\sigma_{2}(f)=\limsup _{r \rightarrow \infty} \frac{\log \log T(r, f)}{\log r} .
$$

In 1976, Rubel and Yang [4] proved the following result.

Theorem 1.1 [4] Let $f$ be a nonconstant entire function. Iff and $f^{\prime}$ share two finite distinct values $C M$, then $f \equiv f^{\prime}$.

In 1996, Brück [5] gave the following conjecture. 
Conjecture 1.1 [5] Let $f$ be a nonconstant entire function. Suppose that $\sigma(f)$ is not a positive integer or infinite, if $f$ and $f^{\prime}$ share one finite value a $C M$, then

$$
\frac{f^{\prime}-a}{f-a}=c
$$

for some nonzero constant c.

In 1998, Gundersen and Yang [6] proved that Brück's conjecture holds for entire functions of finite order and obtained the following result.

Theorem 1.2 [6, Theorem 1] Letf be a nonconstant entire function of finite order. Iff and $f^{\prime}$ share one finite value a $C M$, then $\frac{f^{\prime}-a}{f-a}=c$ for some nonzero constant $c$.

The shared value problems relative to a meromorphic function $f$ and its derivative $f^{(k)}$ have been a more widely studied subtopic of the uniqueness theory of entire and meromorphic functions in the field of complex analysis (see [7-12]).

In 2009, Chang and Zhu [13] further investigated the problem related to Brück's conjecture and proved that Theorem 1.2 remains valid if the value $a$ is replaced by a function.

Theorem 1.3 [13, Theorem 1] Let $f$ be an entire function of finite order and $a(z)$ be a function such that $\sigma(a)<\sigma(f)<\infty$. Iff and $f^{\prime}$ share $a(z) C M$, then $\frac{f^{\prime}-a}{f-a}=$ cfor some nonzero constant c.

Thus, there are natural questions to ask:

(i) What would happen when $\sigma(a)<\sigma(f)<\infty$ is replaced by $0<\sigma(a)=\sigma(f)<\infty$ in Theorem 1.3?

(ii) For Theorems 1.1-1.3, what would happen when $f^{\prime}$ is replaced by differential polynomial

$$
L(f)=a_{k}(z) f^{(k)}+a_{k-1}(z) f^{(k-1)}+\cdots+a_{1}(z) f^{\prime}+a_{0}(z) f
$$

where $a_{k}(z)(\not \equiv 0), \ldots, a_{0}(z)$ are polynomials?

The main purpose of this article is to study the above questions and obtain the following theorems.

Theorem 1.4 Let $f(z)$ and $\alpha(z)$ be two nonconstant entire functions and satisfy $0<\sigma(\alpha)=$ $\sigma(f)<\infty$ and $\tau(f)>\tau(\alpha)$, and $L(f)$ be stated as in (1) such that

$$
\sigma(f)>1+\max \left\{\frac{\operatorname{deg}_{z} a_{j}-\operatorname{deg}_{z} a_{k}}{k-j}, 0\right\} .
$$

Iff $(z)$ and $L(f(z))$ share $\alpha(z) C M$, then

$$
\frac{L(f(z))-\alpha(z)}{f(z)-\alpha(z)}=c
$$

for some nonzero constant c. 
Theorem 1.5 Let $f(z)$ be a nonconstant transcendental entire function with $\sigma_{2}(f)<\infty$, let $\sigma_{2}(f)$ be not an integer, and let $L(f)$ be stated as in (1). Iff and $L(f)$ share a nonzero constant a $C M$ and $\delta(0, f)>0$, then

$$
\frac{L(f(z))-a}{f(z)-a}=c
$$

for some nonzero constant c.

Recently, some papers have studied Brück's conjecture related to difference of entire function (including [14, 15]). In 2009, Heittokangas et al. [14] got the following result.

Theorem 1.6 [14, Theorem 1] Let $f$ be a nonconstant meromorphic function of finite order $\sigma(f)<2$, and let $\eta$ be a nonzero complex number. If $f(z+\eta)$ and $f(z)$ share a finite complex value a $C M$, then $f(z+\eta)-a=c(f(z)-a)$ for all $z \in \mathbb{C}$, where $c$ is some nonzero complex number.

In this paper, we further investigate Brück's conjecture related to entire function and its difference polynomial and obtain the following result.

Theorem 1.7 Let $f(z)$ be a nonconstant entire function of finite order $0<\sigma(f)<\infty, L_{1}(f)$ be difference polynomial off of the form

$$
L_{1}(f(z))=f\left(z+\eta_{k}\right)+f\left(z+\eta_{k-1}\right)+\cdots+f\left(z+\eta_{1}\right)
$$

where $\eta_{k}, \eta_{k-1}, \ldots, \eta_{1}$ are nonzero complex numbers. If $L_{1}(f(z))=c f(z)$ and $\xi(\neq 0)$ is a Borel exceptional value of $f(z)$, then $L_{1}(f)=k f(z)$.

\section{Some lemmas}

To prove our theorems, we will require some lemmas as follows.

Lemma 2.1 [16] Let $f(z)$ be a transcendental entire function, $v(r, f)$ be the central index of $f(z)$. Then there exists a set $E \subset(1,+\infty)$ with finite logarithmic measure, we choose $z$ satisfying $|z|=r \notin[0,1] \cup E$ and $|f(z)|=M(r, f)$, we get

$$
\frac{f^{(j)}(z)}{f(z)}=\left\{\frac{v(r, f)}{z}\right\}^{j}(1+o(1)) \quad \text { for } j \in N .
$$

Lemma 2.2 [17] Let $f(z)$ be an entire function of finite order $\sigma(f)=\sigma<\infty$, and let $v(r, f)$ be the central index of $f$. Then, for any $\varepsilon(>0)$, we have

$$
\limsup _{r \rightarrow \infty} \frac{\log v(r, f)}{\log r}=\sigma .
$$

Lemma 2.3 [18] Letf be a transcendental entire function, and let $E \subset[1,+\infty)$ be a set having finite logarithmic measure. Then there exists $\left\{z_{n}=r_{n} e^{i \theta_{n}}\right\}$ such that $\left|f\left(z_{n}\right)\right|=M\left(r_{n}, f\right)$, $\theta_{n} \in[0,2 \pi), \lim _{n \rightarrow \infty} \theta_{n}=\theta_{0} \in[0,2 \pi), r_{n} \notin E$ and if $0<\sigma(f)<\infty$, then, for any given $\varepsilon>0$ and sufficiently large $r_{n}$,

$$
r_{n}^{\sigma(f)-\varepsilon}<v\left(r_{n}, f\right)<r_{n}^{\sigma(f)+\varepsilon} .
$$


Lemma 2.4 [16] Let $P(z)=b_{n} z^{n}+b_{n-1} z^{n-1}+\cdots+b_{0}$ with $b_{n} \neq 0$ be a polynomial. Then, for every $\varepsilon>0$, there exists $r_{0}>0$ such that for all $r=|z|>r_{0}$ the inequalities

$$
(1-\varepsilon)\left|b_{n}\right| r^{n} \leq|P(z)| \leq(1+\varepsilon)\left|b_{n}\right| r^{n}
$$

hold.

Lemma 2.5 Let $f(z)$ and $A(z)$ be two entire functions with $0<\sigma(f)=\sigma(A)=\sigma<\infty, 0<$ $\tau(A)<\tau(f)<\infty$, then there exists a set $E \subset[1,+\infty)$ that has infinite logarithmic measure such that for all $r \in E$ and a positive number $\kappa>0$, we have

$$
\frac{M(r, A)}{M(r, f)}<\exp \left\{-\kappa r^{\sigma}\right\}
$$

Proof By definition, there exists an increasing sequence $\left\{r_{m}\right\} \rightarrow \infty$ satisfying $\left(1+\frac{1}{m}\right) r_{m}<$ $r_{m+1}$ and

$$
\lim _{m \rightarrow \infty} \frac{\log M\left(r_{m}, f\right)}{r_{m}^{\sigma}}=\tau(f) .
$$

For any given $\beta(\tau(A)<\beta<\tau(f))$, there exists some positive integer $m_{0}$ such that for all $m \geq m_{0}$ and for any given $\varepsilon(0<\varepsilon<\tau(f)-\beta)$, we have

$$
\log M\left(r_{m}, f\right)>(\tau(f)-\varepsilon) r_{m}^{\sigma}
$$

Thus, there exists some positive integer $m_{1}$ such that for all $m \geq m_{1}$, we have

$$
\left(\frac{m}{m+1}\right)^{\sigma}>\frac{\beta}{\tau(f)-\varepsilon} .
$$

From (2)-(4), for all $m \geq m_{2}=\max \left\{m_{0}, m_{1}\right\}$ and for any $r \in\left[r_{m},\left(1+\frac{1}{m}\right) r_{m}\right]$, we have

$$
\begin{aligned}
M(r, f) & \geq M\left(r_{m}, f\right)>\exp \left\{(\tau(f)-\varepsilon) r_{m}^{\sigma}\right\} \\
& \geq \exp \left\{(\tau(f)-\varepsilon)\left(\frac{m}{m+1} r\right)^{\sigma}\right\}>\exp \left\{\beta r^{\sigma}\right\} .
\end{aligned}
$$

Set $E=\bigcup_{m=m_{2}}^{\infty}\left[r_{m},\left(1+\frac{1}{m}\right) r_{m}\right]$, then

$$
m_{l} E=\sum_{m=m_{2}}^{\infty} \int_{r_{m}}^{\left(1+\frac{1}{m}\right) r_{m}} \frac{d t}{t}=\sum_{m=m_{2}}^{\infty} \log \left(1+\frac{1}{m}\right)=\infty .
$$

From the definition of type of entire function, for any sufficiently small $\varepsilon>0$, we have

$$
M(r, A)<\exp \left\{(\tau(A)+\varepsilon) r^{\sigma}\right\}
$$

By (5) and (6), set $\kappa=\beta-\tau(A)-\varepsilon$, for all $r \in E$, we have

$$
\frac{M(r, A)}{M(r, f)}<\exp \left\{-(\beta-\tau(A)-\varepsilon) r^{\sigma}\right\}=e^{-\kappa r^{\sigma}} .
$$

Thus, this completes the proof of this lemma. 
Lemma 2.6 [19, Theorem 2.1] Let $f(z)$ be a meromorphic function of finite order $\sigma$, and let $\eta$ be a fixed nonzero complex number, then, for each $\varepsilon>0$, we have

$$
m\left(r, \frac{f(z+c)}{f(z)}\right)+m\left(r, \frac{f(z)}{f(z+c)}\right)=O\left(r^{\sigma-1+\varepsilon}\right) .
$$

Lemma 2.7 [19, Corollary 2.5] Let $f(z)$ be a meromorphic function with order $\sigma=\sigma(f)$, $\sigma<+\infty$, and let $\eta$ be a fixed nonzero complex number, then, for each $\varepsilon>0$, we have

$$
T(r, f(z+\eta))=T(r, f)+O\left(r^{\sigma-1+\varepsilon}\right)+O(\log r) .
$$

Lemma 2.8 $[1,20]$ Let $g:(0,+\infty) \rightarrow R, h:(0,+\infty) \rightarrow R$ be monotone increasing functions such that $g(r) \leq h(r)$ outside of an exceptional set $E$ with finite linear measure, or $g(r) \leq$ $h(r), r \notin H \cup(0,1]$, where $H \subset(1, \infty)$ is a set of finite logarithmic measure. Then, for any $\alpha>1$, there exists $r_{0}$ such that $g(r) \leq h(\alpha r)$ for all $r \geq r_{0}$.

\section{The proof of Theorem 1.4}

Since $f(z)$ is an entire function, and $f(z)$ and $L(f(z))$ share $\alpha(z) C M$, then there is an entire function $\gamma(z)$ such that

$$
\frac{L(f(z))-\alpha(z)}{f(z)-\alpha(z)}=e^{\gamma(z)}
$$

Next, we will claim that $\gamma(z)$ is a constant.

Suppose that $\gamma(z)$ is transcendental. It follows that $\sigma\left(e^{\gamma(z)}\right)=\infty$. However, since $0<$ $\sigma(f)=\sigma(\alpha)<\infty$, it follows from the left-hand side of (7) that $\sigma\left(\frac{L(f(z))-\alpha(z)}{f(z)-\alpha(z)}\right)<\infty$, a contradiction. Thus, $\gamma(z)$ is not transcendental.

Suppose that $\gamma(z)$ is a nonconstant polynomial, let

$$
\gamma(z)=b_{m} z^{m}+b_{m-1} z^{m-1}+\cdots+b_{0}
$$

where $b_{m}, \ldots, b_{0}$ are constants and $b_{m} \neq 0, m \geq 1$. Thus, it follows from (7) and Lemma 2.4 that

$$
\left|b_{m}\right| r^{m}(1+o(1))=|\gamma(z)|=\left|\log \frac{\frac{L(f(z))}{f(z)}-\frac{\alpha(z)}{f(z)}}{1-\frac{\alpha(z)}{f(z)}}\right| .
$$

Since $L(f)=a_{k} f^{k}+a_{k-1} f^{(k-1)}+\cdots+a_{0} f$, from Lemma 2.1, then there exists a subset $E_{1} \subset$ $(1,+\infty)$ with finite logarithmic measure such that for some point $z=r e^{i \theta}(\theta \in[0,2 \pi))$, $r \notin E_{1}$ and $M(r, f)=|f(z)|$, we have

$$
\frac{f^{(j)}(z)}{f(z)}=\left\{\frac{v(r, f)}{z}\right\}^{j}(1+o(1)), \quad 1 \leq j \leq k .
$$

Thus, it follows that

$$
\begin{aligned}
\frac{L(f(z))}{f(z)} & =a_{k}\left\{\frac{v(r, f)}{z}\right\}^{k}(1+o(1))+\cdots+a_{1}\left\{\frac{v(r, f)}{z}\right\}(1+o(1))+a_{0} \\
& =\frac{a_{k}}{z^{k}}(1+o(1))\left[v(r, f)^{k}+\sum_{j=1}^{k} \frac{a_{k-j}}{a_{k}} z^{j} v(r, f)^{k-j}(1+o(1))\right] .
\end{aligned}
$$


From Lemma 2.3, there exists $\left\{z_{n}=r_{n} e^{i \theta_{n}}\right\}$ such that $\left|f\left(z_{n}\right)\right|=M\left(r_{n}, f\right), \theta_{n} \in[0,2 \pi)$, $\lim _{n \rightarrow \infty} \theta_{n}=\theta_{0} \in[0,2 \pi), r_{n} \notin E_{1}$, then, for any given $\varepsilon$ satisfying

$$
0<\varepsilon<\min _{1 \leq j \leq k} \frac{j \sigma(f)-j-d_{k-j}}{3 k-j},
$$

where $d_{k-j}=\operatorname{deg}_{z} a_{k-j}-\operatorname{deg}_{z} a_{k}$, and sufficiently large $r_{n}$, we have

$$
r_{n}^{\sigma(f)-\varepsilon}<v\left(r_{n}, f\right)<r_{n}^{\sigma(f)+\varepsilon} .
$$

Since $a_{j}(z), j=0,1, \ldots, k$, are polynomials, let $a_{j}(z)=\sum_{t=0}^{s_{j}} l_{j t} z^{t}$, where $s_{j}=\operatorname{deg}_{z} a_{j}, j=$ $0,1, \ldots, k$. Then, from Lemma 2.4 and (10), we have

$$
\begin{aligned}
\left|\frac{a_{k-j}}{a_{k}} z^{j} v(r, f)^{k-j}(1+o(1))\right| & \leq M \frac{\left|l_{k-j, s_{k-j}}\right| r_{n}^{s_{k-j}}}{\left|l_{k, s_{k}}\right| r_{n}^{s_{k}}} r_{n}^{j} r_{n}^{(\sigma(f)+\varepsilon)(k-j)} \\
& =M \frac{\left|l_{k-j, s_{k-j}}\right|}{\left|l_{k, s_{k}}\right|} r_{n}^{d_{k-j+j+(\sigma(f)+\varepsilon)(k-j)}} \\
& \leq M \frac{\left|l_{k-j, s_{k-j}}\right|}{\left|l_{k, s_{k}}\right|} r_{n}^{k \sigma(f)-j \sigma(f)+d_{k-j+j+(k-j) \varepsilon}},
\end{aligned}
$$

where $d_{k-j}=s_{k-j}-s_{k}$ and $M$ is a positive constant. Since $-j \sigma(f)+d_{k-j}+j+(k-j) \varepsilon<-2 k \varepsilon<0$, it follows from (11) that

$$
\left|\frac{a_{k-j}}{a_{k}} z^{j} v(r, f)^{k-j}(1+o(1))\right|<M \frac{\left|l_{k-j, s_{k-j}}\right|}{\left|l_{k, s_{k}}\right|} r_{n}^{k(\sigma(f)-2 \varepsilon)}, \quad r_{n} \notin E_{1} .
$$

Since $0<\sigma(\alpha)=\sigma(f)<\infty$ and $\tau(\alpha)<\tau(f)<\infty$, from Lemma 2.5, there exists a set $E \subset$ $[1,+\infty)$ that has infinite logarithmic measure such that for a sequence $\left\{r_{n}\right\}_{1}^{\infty} \in E_{2}=E-E_{1}$, we have

$$
\frac{M(r, \alpha)}{M(r, f)}<\exp \left\{-\kappa r_{n}^{\sigma(f)}\right\} \rightarrow 0 \quad \text { as } n \rightarrow \infty .
$$

From (8), (9), (12), (13) and Lemma 2.2, we can get that

$$
\left|b_{m}\right| r_{n}^{m}(1+o(1))=|\gamma(z)|=O\left(\log r_{n}\right)
$$

which is impossible. Thus, $\gamma(z)$ is not a polynomial.

Therefore, $\gamma(z)$ is a constant, that is, there exists some nonzero constant $c$ such that

$$
\frac{L(f(z))-\alpha(z)}{f(z)-\alpha(z)}=c .
$$

Thus, this completes the proof of Theorem 1.4.

\section{The proof of Theorem 1.5}

Since $L(f)$ and $f$ share the constant $a C M$, then there exists an entire function $\varphi(z)$ such that

$$
\frac{L(f)-a}{f-a}=e^{\varphi} .
$$


We will consider two cases as follows.

Case 1. If $a=0$, it follows from (15) that

$$
\frac{L(f(z))}{f(z)}=e^{\varphi(z)}
$$

Since $L(f(z))=a_{k}(z) f^{(k)}(z)+\cdots+a_{1}(z) f^{\prime}(z)+a_{0}(z)$ and $a_{j}(z), j=0,1, \ldots, k$, are polynomials, it follows from (16) that

$$
T\left(r, e^{\varphi}\right)=m\left(r, e^{\varphi}\right)=m\left(r, \frac{L(f)}{f}\right) \leq \sum_{j=1}^{k} m\left(r, \frac{f^{(j)}}{f}\right)+\sum_{i=0}^{k} m\left(r, a_{i}\right)=O(\log r T(r, f)),
$$

outside of an exceptional set $E_{3}$ with finite linear measure. Thus, there exists a constant $K$ such that

$$
T\left(r, e^{\varphi}\right) \leq K \log (r T(r, f)) \quad \text { for } r \notin E_{3} .
$$

By Lemma 2.8, there exists an $r_{0}>0$, and for all $r \geq r_{0}$, we have

$$
T\left(r, e^{\varphi}\right) \leq K \log (\zeta r T(\zeta r, f)) \quad \text { for any } \zeta>1
$$

Thus, we can deduce from (17) that $\sigma\left(e^{\varphi}\right) \leq \sigma_{2}(f)<\infty$, that is, $\varphi(z)$ is a polynomial.

By using the same argument as in [21, Theorem 1.1], we can get that $\sigma_{2}(f)=\operatorname{deg}_{z} \varphi$, which is a contradiction to $\sigma_{2}(f)$ is not a positive integer. Thus, $\varphi(z)$ is only a constant, it follows from (15) that $L(f(z))=c f(z)$, where $c$ is a nonzero constant.

Case 2. If $a \neq 0$, from the derivation of (15) and eliminating $e^{\varphi}$, we can get

$$
\varphi^{\prime}(z)=\frac{L^{\prime}(f(z))}{L(f(z))-a}-\frac{f^{\prime}(z)}{f(z)-a} .
$$

If $\varphi^{\prime}(z) \equiv 0$, that is, $\varphi(z) \equiv c, c$ is a constant. Thus, we can prove the conclusion of Theorem 1.5 easily.

If $\varphi^{\prime}(z) \not \equiv 0$, then it follows from (18) that

$$
m\left(r, \varphi^{\prime}\right)=S(r, f)
$$

We can rewrite (18) in the following form:

$$
\begin{aligned}
\varphi^{\prime} & =f\left[\frac{L(f)}{f} \frac{1}{L(f)} \frac{L^{\prime}(f)}{L(f)-a}-\frac{1}{f} \frac{f^{\prime}}{f-a}\right] \\
& =\frac{f}{a}\left[\frac{L(f)}{f} \frac{L^{\prime}(f)}{L(f)-a}-\frac{L^{\prime}(f)}{f}-\frac{f^{\prime}}{f-a}+\frac{f^{\prime}}{f}\right] .
\end{aligned}
$$

Since $\varphi^{\prime} \not \equiv 0$ and $f$ is transcendental, set

$$
\Psi:=\frac{L(f)}{f} \frac{L^{\prime}(f)}{L(f)-a}-\frac{L^{\prime}(f)}{f}-\frac{f^{\prime}}{f-a}+\frac{f^{\prime}}{f}
$$


then we have $m(r, \Psi)=S(r, f)$. Thus, it follows from (20) and (21) that

$$
\frac{a}{f(z)}=\frac{\Psi(z)}{\varphi^{\prime}(z)}
$$

Since $\varphi(z)$ is an entire function, from (18)-(22), then we have

$$
\begin{aligned}
m\left(r, \frac{1}{f}\right) & \leq m(r, \Psi)+m\left(r, \frac{1}{\varphi^{\prime}}\right) \leq S(r, f)+T\left(r, \varphi^{\prime}\right) \\
& =S(r, f)+m\left(r, \varphi^{\prime}\right)=S(r, f) .
\end{aligned}
$$

It follows that

$$
\delta(0, f)=\liminf _{r \rightarrow \infty} \frac{m\left(r, \frac{1}{f}\right)}{T(r, f)}=0,
$$

which is a contradiction to the assumption of Theorem 1.5.

Thus, from Case 1 and Case 2, we complete the proof of Theorem 1.5.

\section{The proof of Theorem 1.7}

Since $f(z)$ is an entire function of finite order $0<\sigma(f)<\infty$ and $\xi(\neq 0)$ is a Borel exceptional value of $f(z)$, then $f(z)$ can be written in the form

$$
f(z)=\xi+p(z) e^{h(z)}
$$

where $h(z)$ is a polynomial of degree $l$ and $p(z)$ is an entire function satisfying $\sigma(p(z))<$ $\sigma(f(z))=\operatorname{deg}_{z} h(z)=l$. Thus, we have

$$
f\left(z+\eta_{j}\right)=\xi+p\left(z+\eta_{j}\right) e^{h\left(z+\eta_{j}\right)}, \quad j=1,2, \ldots, k
$$

From Lemma 2.7, we have $\sigma\left(p\left(z+\eta_{j}\right)\right)<\sigma\left(f\left(z+\eta_{j}\right)\right)=\sigma(f(z))$ and $\operatorname{deg}_{z} h\left(z+\eta_{j}\right)=\operatorname{deg}_{z} h(z)=$ $l$ for $j=1,2, \ldots, k$. Since $L_{1}(f(z))=c f(z)$, it follows from (23) and (24) that

$$
\sum_{j=1}^{k} p\left(z+\eta_{j}\right) e^{h\left(z+\eta_{j}\right)}=(c-k) d+c p(z) e^{h(z)}
$$

Set $h(z)=\mu_{l} z^{l}+\cdots$ and $\mu_{l} \neq 0$, then we can deduce from (25) that

$$
\sum_{j=1}^{k} p\left(z+\eta_{j}\right) e^{\mu_{m-1}^{j} z^{m-1}+\cdots}=\frac{(c-k) d+c p(z) e^{h(z)}}{e^{\mu_{l} z^{l}}} .
$$

Let $\Phi:=\sum_{j=1}^{k} p\left(z+\eta_{j}\right) e^{\mu_{m-1}^{j} z^{m-1}+\cdots}$, it is easy to see that $\Phi \not \equiv 0$ and $\sigma(\Phi)<\sigma(f)$, that is, $T(r, \Phi)=o(T(r, f))=o\left(T\left(r, e^{h(z)}\right)\right)$.

Suppose that $c \neq k$. Since $\xi \neq 0$, it follows from (26) that

$$
N\left(r, \frac{1}{e^{h(z)}-\frac{(c-k) \xi}{c p(z)}}\right)=N\left(r, \frac{1}{\Phi}\right) \leq T(r, \Phi)=S\left(r, e^{h(z)}\right) .
$$


By the second fundamental theorem concerning small functions, for any $\varepsilon>0$, we have

$$
\begin{aligned}
T\left(r, e^{h(z)}\right) & \leq N\left(r, \frac{1}{e^{h(z)}-\frac{(c-k) \xi}{c p(z)}}\right)+\varepsilon T\left(r, e^{h(z)}\right)+S\left(r, e^{h(z)}\right) \\
& =\varepsilon T\left(r, e^{h(z)}\right)+S\left(r, e^{h(z)}\right) .
\end{aligned}
$$

Since $\varepsilon$ is arbitrary, we can get a contradiction from the above inequality. Thus, we can get that $c=k$.

Therefore, we prove that $L_{1}(f(z))=k f(z)$, that is, the conclusion of Theorem 1.7 holds.

\section{Competing interests}

The authors declare that they have no competing interests.

\section{Authors' contributions}

$\mathrm{HW}, \mathrm{L}-\mathrm{ZY}$ and $\mathrm{H}-\mathrm{YX}$ completed the main part of this article, HW, H-YX corrected the main theorems. All authors read and approved the final manuscript.

\section{Author details}

'Department of Informatics and Engineering, Jingdezhen Ceramic Institute, Jingdezhen, Jiangxi 333403, P.R. China.

${ }^{2}$ Department of Mathematics, Shandong University, Jinan, Shandong 250100, P.R. China.

\section{Acknowledgements}

The authors thank the referee for his/her valuable suggestions to improve the present article. The first author was supported by the NSF of China (11301233, 61202313), the Natural Science Foundation of Jiang-Xi Province in China (20132BAB211001), and the Foundation of Education Department of Jiangxi (GJJ14644) of China. The second author was supported by the NSF of China $(11371225,11171013)$.

Received: 24 June 2014 Accepted: 6 October 2014 Published: 27 Oct 2014

\section{References}

1. Hayman, WK: Meromorphic Functions. Clarendon, Oxford (1964)

2. Yang, L: Value Distribution Theory. Springer, Berlin (1993)

3. Yi, HX, Yang, CC: Uniqueness Theory of Meromorphic Functions. Kluwer Academic, Dordrecht (2003); Chinese original: Science Press, Beijing (1995)

4. Rubel, L, Yang, CC: Values shared by an entire function and its derivative. In: Complex Analysis (Proc. Conf., Univ. Kentucky, Lexington, Ky., 1976). Lecture Notes in Mathematics, vol. 599, pp. 101-103. Springer, Berlin (1977)

5. Brück, R: On entire functions which share one value CM with their first derivative. Results Math. 30, 21-24 (1996)

6. Gundersen, GG, Yang, LZ: Entire functions that share one value with one or two of their derivatives. J. Math. Anal. Appl. 223, 85-95 (1998)

7. Mues, E, Steinmetz, N: Meromorphe funktionen, die mit ihrer ersten und zweiten Ableitung einen endlichen Wert teilen. Complex Var. Theory Appl. 6, 51-71 (1986)

8. Zhang, JL, Yang, LZ: A power of a meromorphic function sharing a small function with its derivative. Ann. Acad. Sci. Fenn., Math. 34, 249-260 (2009)

9. Zhang, QC: Meromorphic function that shares one small function with its derivative. J. Inequal. Pure Appl. Math. 6 , Article 116 (2005)

10. $\mathrm{Ai}, \mathrm{LJ}, \mathrm{Yi}, \mathrm{CF}$ : The growth for solutions of a class of higher order linear differential equations with meromorphic coefficients. J. Jiangxi Norm. Univ., Nat. Sci. 38(3), 250-253 (2014)

11. Tu, J, Huang, HX, Xu, HY, Chen, CF: The order and type of meromorphic functions and analytic functions in the unit disc. J. Jiangxi Norm. Univ., Nat. Sci. 37(5), 449-452 (2013)

12. He, J, Zheng, XM: The iterated order of meromorphic solutions of some classes of higher order linear differential equations. J. Jiangxi Norm. Univ., Nat. Sci. 36(6), 584-588 (2012)

13. Chang, JM, Zhu, YZ: Entire functions that share a small function with their derivatives. J. Math. Anal. Appl. 351 491-496 (2009)

14. Heittokangas, J, Korhonen, R, Laine, I, Rieppo, J, Zhang, JL: Value sharing results for shifts of meromorphic functions, and sufficient conditions for periodicity. J. Math. Anal. Appl. 355, 352-363 (2009)

15. Li, XM, Yi, HX: Entire functions sharing an entire function of smaller order with their shifts. Proc. Jpn. Acad., Ser. A, Math. Sci. 89, 34-39 (2013)

16. Laine, I: Nevanlinna Theory and Complex Differential Equations. de Gruyter, Berlin (1993)

17. He, $Y Z, X i a o, X Z$ : Algebroid Functions and Ordinary Differential Equations. Science Press, Beijing (1988)

18. Mao, ZQ: Uniqueness theorems on entire functions and their linear differential polynomials. Results Math. 55 447-456 (2009)

19. Chiang, YM, Feng, SJ: On the Nevanlinna characteristic of $f(z+\eta)$ and difference equations in the complex plane. Ramanujan J. 16, 105-129 (2008)

20. Barnett, DC, Halburd, RG, Korhonen, RJ, Morgan, W: Nevanlinna theory for the q-difference operator and meromorphic solutions of q-difference equations. Proc. R. Soc. Edinb., Sect. A, Math. 137, 457-474 (2007)

21. Li, XM, Yi, HX: An entire function and its derivatives sharing a polynomial. J. Math. Anal. Appl. 330, 66-79 (2007) 
$10.1186 / 1687-1847-2014-274$

Cite this article as: Wang et al.: On some complex differential and difference equations concerning sharing function. Advances in Difference Equations 2014, 2014:274

Submit your manuscript to a SpringerOpen ${ }^{\circ}$ journal and benefit from:

- Convenient online submission

- Rigorous peer review

- Immediate publication on acceptance

Open access: articles freely available online

- High visibility within the field

- Retaining the copyright to your article

Submit your next manuscript at $\boldsymbol{s p r i n g e r o p e n . c o m ~}$ 